\title{
Le parler plurilingue comme ressource d'apprentissage
}

\section{Luci Nussbaum}

\section{(2) OpenEdition \\ Journals}

Édition électronique

URL : http://journals.openedition.org/trema/3269

DOI : $10.4000 /$ trema.3269

ISSN : 2107-0997

Éditeur

Faculté d'Éducation de l'université de Montpellier

Édition imprimée

Date de publication : 1 novembre 2014

Pagination : 76 - 85

ISSN : 1167-315X

Référence électronique

Luci Nussbaum, «Le parler plurilingue comme ressource d'apprentissage », Tréma [En ligne], 42 | 2014 mis en ligne le 16 juin 2015, consulté le 19 avril 2019. URL : http://journals.openedition.org/ trema/3269 ; DOl : 10.4000/trema.3269

Ce document a été généré automatiquement le 19 avril 2019.

Trema 


\title{
Le parler plurilingue comme ressource d'apprentissage
}

\author{
Luci Nussbaum
}

\section{Introduction}

1 L'école du XXIe siècle est, par définition, plurielle et multilingue. Les enfants y arrivent avec des bagages sociolinguistiques divers et ils y apprennent de nouvelles ressources linguistiques, non seulement dans les cours spécifiques (de langues et en langues) mais aussi et notamment avec leurs pairs. L'élargissement de leur répertoire se produit de manière non cloisonnée et souvent par l'utilisation simultanée de ressources appartenant à des systèmes linguistiques divers. Malgré cela, l'institution scolaire - $\mathrm{y}$ compris pour ce qui concerne la formation des enseignants - continue à travailler de manière compartimentée, en proposant des enseignements séparés des langues, sans considérer que, dans leur vie quotidienne, les apprenants sont confrontés à des usages plurilingues ${ }^{1}$, et sans réellement tenir compte de la façon dont les compétences en langues se construisent.

2 L'apprentissage des langues s'initie dans la première enfance et continue tout au long de la vie comme faisant partie des processus de socialisation. La biographie langagière des personnes reflète, à chaque étape, leur contact avec des langues et l'utilisation des variétés linguistiques d'ordre divers. Ces pratiques plurielles procurent des occasions pour acquérir des compétences maximales ou bien des compétences à reconnaître certaines langues, sans pouvoir les utiliser; les personnes peuvent aussi oublier des capacités linguistiques acquises à un moment donné de leur vie (Blommaert \& Bacchus, 2011).

3 Malgré la variabilité des processus d'apprentissage, sous l'influence de certaines conceptions sur les langues et de certaines croyances populaires, il est souvent accepté que l'apprentissage est un processus qui doit suivre des étapes fermées. Ainsi, pour s'initier à l'apprentissage d'une nouvelle langue, faudrait-il avoir "consolidé » l'apprentissage des langues maternelles ou d'autres apprises précédemment. Sans cette 
consolidation, le répertoire de l'apprenant pourrait être atteint par des effets négatifs, comme, par exemple, des mélanges ou des formes hybrides non acceptés par la norme d'une langue donnée. Ceci implique une conception du plurilinguisme comme une addition de compétences étanches en chaque langue et non pas un ensemble de ressources connexes à disposition des personnes à toute fin pratique.

Ces conceptions ont été dépassées par les études qui démontrent que l'apprentissage simultané de plusieurs langues n'est pas nuisible, mais, au contraire, il peut maximiser le développement de compétences à communiquer (García, 2007; García \& Sylvan, 2011; Creese \& Blackledge, 2010 ; Li Wei, 2010 ; Lüdi, 2011 ; Nussbaum, à paraître). L'apprenant de langues n'est pas un individu qui cherche à se rapprocher d'une personne monolingue, native "idéalisée ", mais quelqu'un qui possédera des capacités originales et adaptables aux besoins de chaque moment (Lüdi, 2006). Les approches socioconstructivistes suggèrent que l'apprentissage des langues se produit à partir de la participation à des activités concrètes pour résoudre des tâches concrètes (Mondada \& Pekarek, 2001), ce qui permet non seulement d'accéder aux formes des langues mais aussi et surtout d'acquérir de l'expertise à communiquer (Hall et al., 2006; Masats et al., 2007). Il est évident que, pour pouvoir réaliser une activité, l'apprenant aura recours à l'ensemble de moyens dont il dispose afin de

1. contribuer à la gestion de l'activité (allocation des tours de paroles, proposition de thèmes, etc.) ;

2. formuler des énoncés propres à la réalisation de la tâche et c) résoudre les obstacles de communication.

Dans ce texte, nous nous proposons d'illustrer, premièrement, la manière dont les ressources plurielles interviennent dans les interactions en classe, en même temps qu'elles constituent des outils indispensables dans le processus d'apprentissage. Dans un deuxième moment, nous discuterons certaines orientations qui pourraient inaugurer une nouvelle étape de la didactique des langues.

\section{Trois cas de figure}

6 Dans cette section, nous allons présenter trois cas de pratiques plurilingues enregistrées à l'école primaire en Catalogne. Nous commencerons par une présentation succincte des politiques linguistiques à l'école dans ce contexte.

\section{1. L'éducation langagière en Catalogne}

7 Les lois d'autonomie de la Catalogne établissent la co-officialité de l'espagnol (ou castillan) et du catalan. Afin d'équilibrer son usage social et afin d'assurer sa transmission aux enfants qui n'utilisent pas la langue en famille ou dans leur quotidien, le catalan est la langue véhiculaire de l'école pour la plupart des contenus du curriculum. L'espagnol et une langue étrangère (et parfois deux) sont aussi enseignés, comme matière ou bien comme langue véhiculaire de disciplines non linguistiques. D'après les documents officiels, à la fin de la scolarité obligatoire (6-16 ans), tous les élèves doivent maîtriser les deux langues co-officielles et avoir acquis certaines compétences en langue étrangère. Cette politique d'enseignement des langues doit être adaptée par chaque établissement selon ses caractéristiques sociolinguistiques (population scolarisée, familles, entourage, 
ressources de personnel) et précisée dans son projet linguistique d'établissement (Projecte Lingüístic de Centre), afin de déterminer les actions concernant l'éducation linguistique dans chaque école. Ainsi, par exemple, un établissement peut proposer des classes d'accueil pour les enfants étrangers, l'enseignement d'une deuxième langue étrangère (souvent le français, dans le primaire), l'enseignement d'une matière en langue étrangère, la programmation d'ateliers de renforcement pour certaines langues, l'enseignement extra-scolaire d'une des langues des populations immigrées (arabe, amazigh, chinois, etc.) ou encore la programmation d'activités pour rendre visible la diversité linguistique de l'établissement.

8 Malgré cette volonté d'articuler de manière harmonieuse l'enseignement des langues, dans la plupart des cas, le principe « une langue pour chaque classe » n'est pas mis en question. Par ailleurs, il existe aussi une forte pression de la sauvegarde de la norme de chaque langue, accentuée par le fait que le catalan est considéré une langue en danger à cause d'une longue étape historique de minorisation. De même, certaines forces politiques considèrent que la langue espagnole est en péril à l'école parce qu'elle n'est pas considérée comme une langue véhiculaire.

Notre groupe de recherche travaille depuis un certain nombre d'années, en coopération avec des enseignants, afin d'évaluer les formes de socialisation langagière proposées par l'école et aussi afin d'expérimenter de nouvelles formules pour enseigner les langues ${ }^{2}$. Dans d'autres textes, nous avons présenté le cadre général de notre recherche (Nussbaum, 2008), les enjeux des politiques linguistiques éducatives en Catalogne (Nussbaum, 2006; Codo \& Nussbaum, 2007) ainsi que des expériences pratiques que nous avons menées (Nussbaum \& Rocha, 2008 ; Nussbaum \& Masats, 2012).

10 Ce travail nous a permis de recueillir des données dans les classes « de » langues et « en » langues. Ces données montrent à quel point les situations d'apprentissage sont traversées par la présence de marques transcodiques et de pratiques plurilingues qui sont à la base de la construction de nouvelles compétences. Nous allons par la suite examiner certaines de ces données.

\section{2. En classe d'accueil ${ }^{3}$}

11 Le fragment qui suit est extrait d'une classe d'accueil pour des apprenants du primaire d'origine étrangère. Les enfants sont en train d'écrire au tableau les mots concernant leurs vêtements. Walid (WAL) ne sait pas identifier une des lettres écrites par un des camarades (jaqueta, veste). L'enseignante lui répond et lui rétorque que la lettre n'est pas compréhensible.

12 Fragment $1^{4}$

1. WAL : què lletra es la primera/l (quelle est la première lettre//)

2. ENS : la jota $\backslash$

(le ji $\backslash$ )

3. WAL : es que no se +entinde+ esta lletra\|

(c'est qu'elle ne se distingue pas cette lettre $\backslash$ )

13 Dans les deux énoncés de Walid (ligne 1 et ligne 3 ) il est possible de repérer les marques de trois variétés (espagnol, catalan et darija ou variété de l'arabe parlée au Maroc), faisant partie du répertoire de l'enfant et qui apparaissent sans solution de continuité dans 
l'interaction en classe de catalan. Il faudra un certain temps pour que les enfants arrivent à distinguer ce qui appartient à un système ou à un autre. Mais c'est ce répertoire provisoire qui lui permet de participer dans la petite communauté de la classe. Observons un fragment plus complexe quant à l'utilisation de répertoires composites.

\section{3. En cours de L2 (anglais)}

Fragment 2

Contexte et participants : il s'agit d'une classe d'élèves de 11 ans qui travaillent sur un projet interdisciplinaire (langue anglaise et arts plastiques). Les élèves, en collaboration avec une classe du Canada, devaient créer un e-book à propos de l'amitié entre un peintre catalan et un peintre canadien ${ }^{5}$. Dans le fragment qui suit, trois enfants (Eloi ELO; Juan JOA et Raul, RAU, El2, et l'enseignante, ENS) - et un quatrième qui n'intervient - pas sont autour d'une table en train de compléter une fiche, comme étape préalable à l'écriture d'un texte pour le e-book.

1. ELO : e e $\backslash$ e $\backslash$ a ese $\backslash$ te erre $\backslash$

(l'enfant épelle le mot « easter » et l'écrit en même temps sur la fiche)

2. ENS : okay easter wha: $t / \|$ easter $\backslash \mid$ cross this out easter $\backslash \|$ (okay pâques quoi:// pâques $\backslash$ coche cela pâques $\backslash \|$ )

3. JUA : (il prend la fiche) una creu// (une croix/l)

4. ENS : yes because you used this word $\backslash$ (oui parce que vous avez utilisé ce mot $\backslash \backslash$ )

5. RAU : (à IZA) no : lo hago yo:o\| (non c'est moi qui le fait $\backslash$ )

6. JUA : yo que no he hecho nada \| (moi qui n'ai rien fait $\backslash$ )

7. RAU : vale $\backslash \|$ (d'accord $\backslash$ )

8. ENS : okay $\backslash$ easte:er $\backslash$ easter street// easter house/II (okay $\backslash$ pâques : $\backslash$ rue pâques/| maison pâques/|

9. RAU : +ihla $+\backslash$ (île\) (prononcé avec aspiration du s)

10. ENS : yes read it $\backslash$ easter $\backslash$ (oui lis-le $\backslash$ easter $\backslash$ )

11. RAU : +island $+\backslash \mid$

(île $\backslash)$ (prononcé en anglais avec toutes ses lettres)

12. ENS : island very good $\backslash$

(île très bien $\backslash$ )

13. RAU : (il prend la fiche) ya escribo yo porque no $x x x$ (j'écris moi parce que je ne $\mathrm{xxx}$ )

14. ENS : okay \\|

(d'accord $\backslash \|$ )

15. ELO : i: $\backslash$

(i :)

16. ENS : no no no they are in easter here you write here in easte:r $\backslash$ (no no no ils sont à pâques ici écrivez ici à pâques:\) 
17. RAU : goma: \|

(gomme $\backslash$ )

18. ENS : rubber $: \backslash$

(gomme $\backslash$ )

19. ELO : xxx

20. RAU : thank you: $\backslash$

(merci: $\backslash)$

21. ENS : (avec un ton fort de surprise et en souriant) welcome $\backslash$

(pas de quoi $\backslash$ )

22. ENS : what's your name// what's your name//

(comment tu t'appelles// comment tu t'appelles/|)

23. JOA : juan $\backslash$

24. ENS : juan \}

25. ENS : can you cross the word island/| in the paper $\backslash \mid$ cross it out $\backslash \mid$ (peux-tu cocher le mot île// sur le papier $\backslash$ coche-le $\backslash$

26. ENS : juan juan ju :an \\|

27. ELO : easter island $\backslash$

(île de pâques)

28. ENS : easter island $\backslash$ yes very good $\backslash \mid$

(île de pâques $\backslash$ oui très bien $\backslash$ )

29. JUA : tatxo//

(je biffe/l)

30. ENS : yes $\backslash$ cross it out $\backslash$

(oui $\backslash \mid$ coche-le)

Le fragment montre un ensemble de pratiques complexes. À la ligne 1, Juan écrit un mot sur la fiche en l'épelant en espagnol. L'enseignante (ligne 2) veut que l'enfant complète l'énoncé, mais, sans attendre la réponse, elle demande à Juan de cocher le mot dans la liste qui figure sur la fiche. Juan demande confirmation de l'ordre en s'adressant en catalan à l'enseignante ; celle-ci justifie sa demande (lignes 3 et 4). Dans les lignes 5 et 6 s'établit un échange, en espagnol, entre Raul et Juan pour décider qui écrit sur la fiche. L'enseignante (ligne 8) revient ensuite sur la question entamée en ligne 2 . Raul répond en espagnol et l'enseignante confirme et demande de lire, chose qui est faite par Raul en prononçant toutes les lettres; l'enseignante accepte en répétant le mot en anglais standard (lignes 9 et 12). Raul demande à écrire, l'enseignante accepte et l'enfant écrit, mais l'enseignante n'accepte pas ce qu'il a écrit (lignes 13 et 16). Alors Raul demande la gomme en espagnol, l'enseignante lui la donne ; mais celle-ci reprend le mot en le disant en anglais. Eloi semble dire à Raul de remercier l'enseignante, ce que celui-ci fait en anglais; l'enseignante répond en souriant (lignes 17 et 21). En ligne 22, l'enseignante demande son nom à Juan, celui-ci répond ; l'enseignante lui demande de cocher le mot ; mais c'est Eloi qui essaye de le faire. L'enseignante veut que ce soit fait par Juan. Finalement c'est Eloi qui formule en anglais l'énoncé entier que demande l'enseignante depuis la ligne 2 (easter island) ; elle accepte (lignes 22 et 27).

Ce fragment illustre plusieurs phénomènes quant à la gestion et l'utilisation des langues. Premièrement, il faut noter que les élèves utilisent l'espagnol pour interagir entre eux (lignes 5-7, 13, 17), tandis qu'ils emploient le catalan pour s'adresser à l'enseignante (lignes 3, 29). Ceci indique les processus de socialisation auxquels nous avons fait 
allusion: les enfants choisissent la langue en fonction du destinataire, comportement habituel en Catalogne. Cependant, ce qui nous intéresse est le fait que les apprenants réussissent à formuler l'énoncé objet de travail en langue cible après un ensemble d'essais - ihla fi island fi i : fi easter island.

C'est un long travail à l'oral et à l'écrit qui se fait de manière conjointe et grâce au concours des ressources dont disposent les apprenants. Ces pratiques plurilingues dans les premières phases de l'apprentissage peuvent aboutir à des pratiques qui s'orientent vers des modes unilingues, comme nous l'observons dans le fragment suivant.

\section{4. En cours en L2 (français)}

Dans ce cas, il s'agit d'un cours de sciences sociales en français avec des élèves du primaire qui suivent cet enseignement depuis trois ans. Dans l'activité, les élèves doivent parler des faits qui les ont intéressés après une lecture proposée par l'enseignante (ENS) Albert (ALB) demande à parler sur une partie de la lecture ; un autre élève, Matias (MAT) intervient, ainsi que plusieurs apprenants (EEE) de manière simultanée.

19 Fragment $3^{6}$

1. ALB : je peux dire choses de le : deuxième//

2. ENS : oui \ de la deuxième feuille $\backslash$ pourquoi pas//

3. ALB : ils men_ ai_| à le temps préhistorique-| ils mangent la viande +crude $+\backslash \mid$

4. ENS : oui $\backslash$ et nous est-ce qu'on mange la viande crue//

5. ALB : nous mange la viande- (des gestes)

6. ENS : cuite cuite $\mid$

7. MAT : fregida al forn $\backslash$ (cuite au four)

8. ENS : cuite cuite $\backslash$ on cuit la viande $\backslash$ nous la cuissons $\backslash$ mais qu'est-ce-que-| de quoi nous avons besoin pour cuire la viande//

9. EEE : le feu\I

10. ENS : du feu hein// et est-ce qu'on connaissait le feu au temps préhistorique//

Le fragment présente une interaction fluide, avec la participation spontanée des apprenants, qui s'orientent vers un mode unilingue d'interaction, qui laisse voir, cependant, des traces de ressources plurilingues dans la morphosyntaxe (lignes 1, 3 et 5) et le lexique (lignes 3,7 ). La proposition du mot inventé « crude » déclenche une séquence de travail sur la forme qui enchaîne avec l'activité centrale en cours.

Les fragments présentés mènent à une reconsidération des conceptions sur la construction des compétences et d'une manière générale sur la didactique des langues à l'école. Nous allons discuter ces aspects dans la section suivante.

\section{Sociolinguistique, apprentissage et didactique des langues}

Pendant des décennies, des études sur les usages plurilingues en contexte social multilingue et des études sur l'apprentissage des L2 ont parcouru des chemins parallèles, avec peu de moments de confluence. Cependant, on peut citer, premièrement, l'utilisation de certains concepts de la sociolinguistique du contact de langues 
(interférence, emprunt, par exemple) pour caractériser l'interlangue des apprenants; deuxièmement, dans le cadre de la sociolinguistique variationniste, le répertoire de l'apprenant est compris comme étant un dialecte dynamique, soumis à des évolutions et aussi à des adaptations au contexte et aux interlocuteurs.

Une partie de la sociolinguistique suisse (voir Lüdi \& Py, 1986 [2003] ; Lüdi \& Py, 2009, comme auteurs pionniers) est sans doute une des premières à articuler dans son programme de travail trois phénomènes: le contact des langues, l'interaction et l'apprentissage, en réussissant à intégrer des perspectives étiques (les observations des chercheurs sur les données analysées) et les perspectives émiques (la catégorisation, directe ou indirecte, des locuteurs sur leurs productions). L'observation des interactions entre les locuteurs novices et experts - en classe ou hors classe - permet de déterminer la façon dont les personnes utilisent les différents microsystèmes linguistiques en présence à partir de procédures qui fournissent des indices sur les processus d'apprentissage de nouvelles ressources langagières. Dans son souci de capter la socialisation langagière dans les interactions, cette sociolinguistique explore la manière dont l'apprenant - personne bi/plurilingue en construction ou en voie de le devenir communicativement experte construit et reconstruit, avec l'aide de ses partenaires, son répertoire verbal.

Le continuum entre utilisation et apprentissage des langues est d'autant plus évident en ce moment même (entre deux siècles), caractérisé par les mouvements de populations très diverses et par les innovations en technologies de la communication. Ces phénomènes posent au premier plan le plurilinguisme dans la vie quotidienne et dans les institutions éducatives et obligent à inclure dans un même paradigme compréhensif l'utilisation et l'apprentissage des langues en contextes multilingues.

Le parler bilingue ou le plurilanguaging (Lüdi, 2011) inclut aussi bien les alternances de langue, les traductions, mais aussi des formes hybrides créées ad hoc par les interlocuteurs à toutes fins pratiques ${ }^{7}$. Comme le signalent García et Sylvan (2011), ces formes de communication ne sont pas seulement des pratiques servant à étayer l'apprentissage, mais elles font aussi partie des régimes discursifs avec lesquels les apprenants du XXIe siècle doivent se familiariser puisqu'ils sont fréquents dans les contextes de globalisation (interactions avec des personnes d'autres cultures, communication via Internet, etc.).

Dans notre travail collaboratif avec des enseignants du primaire nous proposons des séquences didactiques dans lesquelles le plurilinguisme est non seulement admis dans l'interaction en classe, mais encouragé à partir de l'utilisation de documents oraux, écrits et audiovisuels en plusieurs langues (Nussbaum \& Rocha, 2008; Nussbaum, 2013). L'approche par projets motive les apprenants à la construction d'un produit final (oral, écrit, audiovisuel) unilingue en langue cible ; mais le processus qui permet de le créer est tout naturellement plurilingue. C'est une démarche semblable à celle qui est proposée par García et Sylvan (2011) et par Duverger (2007) lorsque cet auteur encourage à « didactiser » le plurilinguisme en classe.

\section{Remarque finale}

Dans les premières étapes de l'apprentissage, les élèves peuvent participer à des activités en langue cible en ayant recours au parler plurilingue, tel qu'il est illustré dans le fragment 2. Ces ressources plurielles constituent l'étayage permettant de participer à des 
tâches complexes. L'apprentissage d'une langue supposerait donc être capable de passer d'un mode plurilingue à un mode unilingue, si besoin est. Les fragments 1 et 3 montrent des moments dans lesquels les apprenants interviennent en s'orientant clairement vers ce type de pratiques.

Ainsi, de notre point de vue, une didactique des langues devrait considérer les pratiques plurilingues - les alternances de langues, les recours à des formes hybrides, par exemple comme un atout permettant aux apprenants leur participation à la diversité d'activités de classe et le chemin incontournable pour acquérir d'autres ressources en langue cible, comme nous avons essayé de l'illustrer à partir de quelques fragments d'interaction. Mais «didactiser le plurilinguisme» (Duverger, 2007) suppose aussi ne pas craindre l'utilisation de plusieurs langues tout au long d'une même séquence didactique, à travers la lecture, la consultation de documents en ligne ou la production écrite ou orale. Ces activités translinguistiques rapprochent la classe des activités quotidiennes des apprenants.

Annexe : Symboles utilisés dans les transcriptions

\begin{tabular}{|l|l|}
\hline Initiales du nom & Majuscules \\
\hline Intonation ascendante, descendante maintenue & I I - \\
\hline Pauses moins et plus longues & $\mathrm{I} \| \mathrm{II}$ \\
\hline Rallongement syllabique selon durée & $::::$ \\
\hline Chevauchement & {$[$ texte] } \\
\hline Interruption & texte- \\
\hline Commentaires et traduction & (commentaires) \\
\hline Formes hybrides & + texte+ \\
\hline Fragments incompréhensibles & xxx \\
\hline Anglais & caractères italiques \\
\hline Espagnol & caractères gras \\
\hline Catalan & caractères soulignés \\
\hline
\end{tabular}

\section{BIBLIOGRAPHIE}

Blommaert, J. \& Bacchus, A. (2011). Repertoires revisited : « Knowing language » in superdiversity. Working Papers in Urban Language \& Literacies, 67.

Codo, E. \& Nussbaum, L. (2007). Plurilinguisme et promotion d'une langue étrangère. Langage et société, 121-122, 275-288.

Creese, A. \& Blackledge, A. (2010). Translanguaging in the bilingual classroom : A pedagogy for learning and teaching ? The Modern Language Journal 94, 103-115.

Duverger, J. (2007). Didactiser l'alternance des langues en cours de DNL. Tréma, 28 [en ligne : http://trema.revues.org/302]. 
García, O. \& Sylvan, C. (2011). Pedagogies and Practices in Multilingual Classrooms: Singularities in Pluralities. The Modern Language Journal, 95, 385-400.

Hall, J., Cheng A. \& Carlson, M. (2006). Reconceptualizing Multicompetence as a Theory of Language Knowledge. Applied Linguistics, 27(2), 220-240.

Li, W. (2010). Voices from the diaspora : Changing hierarchies and dynamics of Chinese multilingualism. International Journal of the Sociology of Language, 205, 155-171.

Lüdi G. (2006). De la compétence linguistique au répertoire plurilingue. Bulletin suisse de linguistique appliquée 84, 173-189.

Lüdi, G. (2011). Vers de nouvelles approches théoriques du langage et du plurilinguisme. Travaux neuchâtelois de linguistique, 53, 47-64.

Lüdi, G. \& Py, B. (2001). Être bilingüe. Berne : Peter Lang.

Masats, D., Nussbaum, L. \& Unamuno V. (2007). When activity shapes the repertoire of second language learners. EUROSLA Yearbook ,7, 121-247.

Mondada, L. \& Pekarek, S. (2001). Interactions acquisitionnelles en contexte: perspectives théoriques et enjeux didactiques. Le Français dans le Monde, 107-142.

Nussbaum, L. (2006). Les défis de l'école pour le maintien et la transmission du catalan. Études de Linguistique Appliquée, 143, 355-370.

Nussbaum, L. (2008). Construire le plurilinguisme à l'école: de la recherche à l'intervention et de l'intervention à la recherche. In Candelier, M. ; Ionnitou, G. ; Omer, D. \& Vasseur, M.-T. (dirs), Conscience du plurilinguisme. Rennes : Presses Universitaires, pp.125-144.

Nussbaum L. (2013). Interrogations didactiques sur l'éducation plurilingue. In Bigot, Bretegnier A. \& Vasseur M.-T. (eds.) Vers le plurilinguisme? 20 ans après. Paris : Albin Michel, pp. 85-94.

Nussbaum L. \& Rocha P. (2008). L'organisation sociale de l'apprentissage dans une approche par projet. Babylonia, 3, 2-55.

Nussbaum L. \& Masats D. (2012). Socialisation langagière en Catalogne : le mutilinguisme comme étayage de pratiques monolingües. In M. Dreyfus et J.-M. Prieurs (dirs.), Hétérogénéité et variation. Perspectives socolinguistiques, didactiques et anthropologiques. Paris : Michel Houdiard éditeur, pp. 155-167.

\section{NOTES}

1. Dans ce texte, nous adoptons le qualificatif «multilingue » pour nous référer aux pays, zones géographiques et institutions dans lesquels plusieurs langues sont co-officielles. Le qualificatif "plurilingue » est appliqué ici, d'une part, à la compétence des personnes et, d'autre part, aux interactions dans lesquelles des ressources provenant de systèmes linguistiques différents sont utilisées.

2. Démarches et matériels construits conjointement avec les enseignants peuvent être consultés sur le site http//:greip.uab.cat

3. Ces données ont été recueillies par Elisabeth Plaza.

4. Voir les symboles transcription en annexe.

5. Voir le site http://pagines.uab.cat/pads/ pour plus de détails à propos de l'expérience et du projet financé par le Ministerio de Ciencia e Innovación (EDU2010-17859).

6. Ces données ont été recueillies par Àngels Campà. 
7. D'autres auteurs utilisent des termes tels que languaging et translanguaging pour se référer à ces phénomènes (voir García \& Sylvan, 2011 ; Nussbaum, à paraître).

\section{RÉSUMÉS}

L'école actuelle est plurielle et multilingue. Les enfants y arrivent avec des bagages sociolinguistiques divers; leur répertoire s'élargit souvent par l'utilisation simultanée de ressources appartenant à des systèmes linguistiques divers. Mais l'école propose l'enseignement séparé des langues, sans accorder de réel intérêt ce type de pratiques. Dans ce texte, nous illustrons la manière dont les ressources plurielles interviennent dans les interactions en classe et leur rôle indispensable dans le processus d'apprentissage. Nous discutons aussi certaines orientations qui pourraient inaugurer une nouvelle étape de la didactique des langues.

The present-day school is plural and multilingual. Children arrive with various sociolinguistic backgrounds, often expanding their repertoire by the simultaneous use of resources belonging to different linguistic systems. But school teaches languages in a separate way, without considering plurilingual practices. In this paper, we illustrate how the plural resources intervene in classroom interactions and their essential role in the learning process. We also discuss some new orientations which could guide a new stage of language teaching.

\section{INDEX}

Mots-clés : apprentissage de langues, didactique, plurilinguisme

Keywords : language learning, language teaching, plurilingualism

\section{AUTEUR}

\section{LUCI NUSSBAUM}

Professeura, Universitat Autònoma de Barcelona, Grup de Recerca en Ensenyament i Interaccions Plurilingües (GREIP) 